DOI: 10.1038/s41467-018-07156-3

\title{
Author Correction: Larger mammals have longer faces because of size-related constraints on skull form
}

Andrea Cardini ${ }^{1,2,3,4} \&$ P. David Polly ${ }^{5}$

Correction to: Nature Communications; https://doi.org/10.1038/ncomms3458; published online 18 September 2013.

In Table 1 of this Article, the descriptions of landmarks 14, 15, and 36 are incorrect. Landmarks 14 and 36 should read "Posterior extremity of occipital condyle along margin of foramen magnum" and landmark 15 should read "Opisthion". A correct version of Table 1 appears below; the error has not been fixed in the original Article.

Published online: 06 November 2018

\footnotetext{
${ }^{1}$ Dipartimento di Scienze Chimiche e Geologiche, Universita` di Modena e Reggio Emilia, I.go S. Eufemia 19, 41121 Modena, Italy. ${ }^{2}$ Centre for Anatomical and Human Sciences, University of Hull, Cottingham Road, Hull HU6 7RX, UK. ${ }^{3}$ Center for Anatomical and Human Sciences, University of York, Heslington, York YO10 5DD, UK. ${ }^{4}$ Centre for Forensic Science, The University of Western Australia, 35 Stirling Highway, Crawley, WA 6009, Australia. ${ }^{5}$ Department of Geological Sciences, Indiana University, 1001 East 10th Street, Bloomington, Indiana 47405, USA. Correspondence and requests for materials should be addressed to A.C. (email: andrea.cardini@unimore.it)
} 


\section{Table 1 Landmark description}

\section{Landmark Description}

$1 \quad$ Prosthion: antero-inferior point on projection of pre-maxilla between central incisors

2, 27 Maxilla: anterior extreme of tooth row (before first premolar)

3, 28 Maxilla: contact points between adjacent pre-molars/ molars, projected labially onto alveolar margin

4, 29 Maxilla: contact points between adjacent pre-molars/ molars, projected lingually onto alveolar margin

5, 30 Maxilla: posterior midpoint onto alveolar margin of last molar

6 Palatine: posterior edge on the midline

7 Basioccipital and basisphenoid: meeting point along midline

8, 31 Basioccipital, basisphenoid and tympanic bulla: meeting

9, $32 \quad$ Foint

10, 33 Anterior tip of acoustic meatus

11, $34 \quad$ Posterior tip of acoustic meatus

12, 35 Posterior tip of jugular foramen

13

14,36

Basion: anterior-most point of foramen magnum

Posterior extremity of occipital condyle along margin of foramen magnum

15 Opisthion

16, 37 Tip of paraoccipital process

17, 38 Infraorbital foramen (center on side external to the orbit)

18, 39 Lacrimal foramen

19, $40 \quad$ Optic foramen

20, $41 \quad$ Zygo-temp inferior: infero-lateral point of zygomatico (jugal) - temporal (squamosal) suture on lateral face of zygomatic arch

$21 \quad$ Rhinion: most anterior midline point on nasals

22, 42 Nasal And pre-maxilla: meeting point on margin of piriform aperture

23, 43 Maxilla and premaxilla: meeting point on margin of palate

$24 \quad$ Nasion: midline point on fronto-nasal suture

25, $44 \quad$ Notch anterior to postorbital process

26 Inion: most posterior point of the cranium 